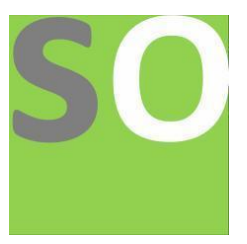

Article title: On a Heuristic Viewpoint Concerning the Quantized Space-time Authors: Yuchen Liu[1]

Affiliations: Shenzhen University[1]

Orcid ids: 0000-0002-6517-0022[1]

Contact e-mail: liuyuchenmdcg@163.com

License information: This work has been published open access under Creative Commons Attribution License $\mathrm{http}: / / c r e a t i v e c o m m o n s . o r g / l i c e n s e s / b y / 4.0 /$, which permits unrestricted use, distribution, and reproduction in any medium, provided the original work is properly cited. Conditions, terms of use and publishing policy can be found at https://www.scienceopen.com/.

Preprint statement: This article is a preprint and has not been peer-reviewed, under consideration and submitted to ScienceOpen Preprints for open peer review.

DOI: 10.14293/S2199-1006.1.SOR-.PPHKL3X.v1

Preprint first posted online: 23 June 2020

Keywords: theoretical physics, relativity, quantized space-time, dark matter, dark energy, universe evolution 


\title{
On a Heuristic Viewpoint Concerning the Quantized Space-time
}

\author{
Yuchen $\mathrm{Liu}^{* 1}$ \\ The First Affiliated Hospital of Shenzhen University, Shenzhen 518035, China \\ *Correspondences: Yuchen Liu (liuyuchenmdcg@163.com).
}

\begin{abstract}
Einstein's theory of relativity revolutionized the view of space-time in physics, and its basic inferences have been continuously verified in the past 100 years. However, the theory of relativity is built on the basis of continuous and smooth space-time, and it cannot be compatible with the concept of discrete space-time in quantum mechanics, which causes many difficulties in the development of modern physics. This paper presents for the first time a heuristic viewpoint in which space-time is considered to be a type of quantized waves propagating at the speed of light. Based on this concept, it re-explains many inferences of special relativity and general relativity, proposes new theory on gravitation and space-time, and provides a preliminary unified framework for explaining dark matter, dark energy, gamma-ray bursts, high energy emission mechanisms in quasar, the origin and evolution of the universe and other difficult problems in modern physics.
\end{abstract}




\section{Introduction}

In 1905, Albert Einstein published the theory of special relativity based on the principle of relativity and the assumption of constant speed of light [1]. The constants "space", "time", and "mass" in Newton's theoretical system were regarded as physical variables related to the speed of movement. In 1909, Minkowski further pointed out that the three-dimensional space and the one-dimensional time should be integrated into a four-dimensional space-time, and the speed of movement of objects in space-time is a constant and the same as the speed of light [2]. In order to extend the principle of relativity from the inertial reference frame to the both inertial and non-inertial reference frames and to explain gravitation, Einstein published the theory of general relativity in 1916 [3], and pointed out that gravitation is a geometric effect produced by the curved space-time around an object. This theory provided a reasonable explanation for the abnormal perihelion precession phenomenon of mercury, and gave important inferences about the gravitational deflection of light, the gravitational redshift of light, the gravitational time dilation, and the gravitational waves. General relativity also provided a solid foundation for modern theories on black hole [4] and the Big Bang [5]. Its main conclusions have been supported by many observational evidences over the past 100 years. Einstein's relative view of space-time revolutionized physics and completely replaced Newton's viewpoint of absolute space and time.

However, general relativity is not a complete theory. Firstly, its gravitational field equation contains 10 second-order nonlinear differential sub-equations, which is very difficult to get an exact solution. Secondly, this theory is not compatible with quantum mechanics [6], which describes the microcosmic world. The geometric gravity cannot be unified with the other three quantized basic forces, including strong force, weak force and electromagnetic force. Thirdly, the theory predicts the existence of space-time singularities but also fails at the singularities. Finally, the theory cannot deal with "dark matter"[7] and "dark energy" [8], the most important research issues in the modern physics. The above contradictions are not inherent in nature, but indicate that the theory needs to be further improved.

The crux of general relativity lies in its fundamental belief that space-time are continuous and smooth, while quantum mechanics, represented by loop quantum theory [9], believes that space may be composed of numerous discrete particles. Therefore, it is urgent to establish a new space-time theory (quantum gravity theory) to unify the seemingly incompatible general relativity and quantum mechanics.

According to special relativity [1], the velocity of any object in space-time is a constant and the same as the speed of light; Based on general relativity [3], gravitational fields have energy and travel at the speed of light, and the essence of gravitational fields is the curving of space-time; On the basis of quantum mechanics [6], the energy transmission is characterized by quantization in microscopic area. From the above three basic points, we seem to be able to get a heuristic viewpoint: space-time has both wave and quantization characteristics, and is composed of space-time quanta that move at the speed of light and propagate in a wave-like manner. In particular, space-time is discrete under the Planck scale and has a minimum value which was contained in a space-time quantum. A space-time quantum is essentially equivalent to a point on 
the world line of space-time in special relativity. A discrete number of space-time quanta moves at the speed of light and travels outward as waves from the source of radiation. Space-time can only vary by integer multiples of space-time quanta, not in the form of continuous smoothing. The quantized nature of energy may result from the microscopic discontinuity of space-time. In other words, the physical world is essentially composed of space-time quanta with high frequencies of vibration. The frequency of space-time wave is proportional to the energy of a space-time quantum.

\section{Basic description of theory on quantized space-time waves}

Consistent with the theory of loop quantum gravity [9], we believe that there exists a minimum non-zero scale in one dimensional space, which is about a Planck length $1_{p}$. Similarly, there is a minimum non-zero scale of time, which is approximately a Planck time $t_{p}$. The difference is that the theory on quantized space-time waves believes that a space-time quantum moves at the speed of light and propagates outward from the radiation source in the form of waves. Therefore, the velocity, time, and space measurements of each space-time quantum in the one-dimensional coordinate system are all constants, which are $c, t_{p}, l_{p}$ respectively. The mathematical relationship between them is $\mathrm{c}=\frac{\mathrm{l}_{\mathrm{p}}}{\mathrm{t}_{\mathrm{p}}}$, where $\mathrm{c}$ is the speed of light in vacuum. The "instant" of the space-time moves at the speed of light, and the emission frequency (k) of the space-time quanta is equivalent to the speed of time flow described in relativity theory. If there is only one object in the universe, the four-dimensional space-time around the object is generated by the space-time quanta radiated continuously by the radiation source. Since all the elementary units that make up the object emit space-time quanta in different directions, this phenomenon can be equivalently regarded as radiating gravitational energy outward from the radiation source (the object) in the form of spherical waves.

Because the speed of a space-time quantum is equal to the speed of light, it can be concluded that the light quantum is a special kind of space-time quantum. According to classical electromagnetism theory, the momentum (p) transmitted by electromagnetic wave is equal to E/ C, while the momentum (p) transmitted by electromagnetic wave is also equal to mc according to classical mechanics. Based on the two equations, the energy of a light quantum is determined by $\mathrm{E}=\mathrm{mc}^{2}$. At the same time, according to the quantum theory, the formula for calculating the energy of a light quantum is: $\mathrm{E}=\mathrm{h} v$.

By extending the above situation to a general situation, it can be concluded that the mathematical relationship between the energy (E) of a space-time quantum and the mass (m) of its corresponding elementary particle is: $\mathrm{E}=\mathrm{mc}^{2}$, which is the mass-energy relationship of special relativity. The mathematical relationship between the natural inherent frequency (v) of a space-time quantum and the energy (E) of a space-time quantum is: $E=h v$, which is the same as the energy calculation formula of the light quantum. So the frequency (v) of a space-time quantum determines the mass-energy of an elementary particle. Since there is a gravitational field that 
propagates at the speed of light around the mass of a substance according to general relativity, the graviton described in quantum theory is essentially the same as the space-time quantum. Therefore, the energy of the gravitational field (the energy of a space-time quantum) generated instantaneously around the elementary particle is $\mathrm{mc}^{2}$. The energy of the gravitational field produced by any object composed of the elementary particles satisfies this relationship, which is the same as the someone's calculation based on general relativity [10]. So the mathematical formula describing the total energy (E) of an object in space-time is as follows:

\section{$\mathrm{E}=\mathrm{Nmc}^{2}=\mathrm{Nhv}$,}

where $\mathrm{N}$ is the total number of space-time quanta, whose value depends on the existence time (life) of the object, $v$ is the total frequency of the space-time waves. Therefore, the mass-energy formula in special relativity only describes the total energy of an object at a specific time point.

At the same time, we should also consider an important property of space-time. Because the total energy of an object in space-time is certain (energy conservation), the product of the natural frequency ( $v$ ) of the time-space waves released by the radiation source and its emission frequency (k) is a certain value in a unit time of the observer. In other words, the natural frequency of time-space waves is larger when the speed of time flow is slowed down, otherwise it is smaller. The mathematical expression of the above process:

$$
\mathrm{E}_{1}=\mathrm{k}_{1} \mathrm{hu}_{1}=\mathrm{E}_{2}=\mathrm{k}_{2} \mathrm{hu}_{2} \text {, so } \mathrm{k}_{1} \mathrm{v}_{1}=\mathrm{k}_{2} \mathrm{v}_{2} .
$$

\section{Reconstructing special relativity by considering the view of quantized space-time waves}

The basic hypothesis of special relativity is that "the speed of light is a constant and does not depend on the inertial reference system". This hypothesis can be deduced from Maxwell's equations and verified by the Michelson-Morley experiment.

Based on the theory of quantized space-time waves described above, it is not difficult to find that the basic property of "constant speed of light" is determined by the quantization characteristics of space-time. No matter which reference system is chosen, the number $(\mathrm{N})$ of space-time quanta obtained by the observer is an integer. So the speed of the photon is:

$\mathrm{v}=\mathrm{s} / \mathrm{t}=\mathrm{N} 1_{\mathrm{p}} / \mathrm{N} \mathrm{t}_{\mathrm{p}}=1_{\mathrm{p}} / \mathrm{t}_{\mathrm{p}}=\mathrm{c}$,

Therefore, the speed of light in vacuum is a constant value, which has no relationship with the reference system chosen by the observer.

Lorentz introduced Lorentz transformation [11] to deal with the premise of "constant speed of light". It is believed that the ruler will contract in its direction of motion, and the basic formula describing this process is: $\mathrm{l}=\mathrm{l}_{0} \sqrt{1-\left(\frac{\mathrm{V}}{\mathrm{c}}\right)^{2}}$, where $\mathrm{l}_{0}$ is the static length of the ruler, $\mathrm{l}$ is 
the moving length of the ruler, $\mathrm{v}$ is the moving speed of the ruler, and $\mathrm{c}$ is the speed of light in vacuum.

From the perspective of quantized space-time waves, it is clear to see that the shortened length of the ruler in the direction of motion can be understood as the reduction in the emission frequency (k) of space-time quanta, as demonstrated in the following formula:

$$
\mathrm{l}=\mathrm{kl}_{\mathrm{p}}=\mathrm{k}_{0} \mathrm{l}_{\mathrm{p}} \sqrt{1-\left(\frac{\mathrm{V}}{\mathrm{c}}\right)^{2}}
$$

Among them, $\mathrm{k}_{0}$ is the emission frequency of space-time quanta when the ruler is stationary, $\mathrm{l}_{\mathrm{p}}$ is the Planck length, $v$ is the velocity of the ruler, and $\mathrm{c}$ is the speed of light in vacuum.

The above formula can be simplified as follows: $\mathrm{k}=\mathrm{k}_{0} \sqrt{1-\left(\frac{\mathrm{v}}{\mathrm{c}}\right)^{2}}$.

The meaning of the formula can be expressed as that the motion of an object is essentially equivalent to the reduction of emission frequency $(\mathrm{k})$ of the space-time quanta in the direction of motion. Similarly, we can get the formula for describing the time expansion effect of special relativity:

$\mathrm{kt}_{\mathrm{p}}=\mathrm{t}=\mathrm{k}_{0} \mathrm{t}_{\mathrm{p}} \sqrt{1-\left(\frac{\mathrm{v}}{\mathrm{c}}\right)^{2}}=\mathrm{t}_{0} \sqrt{1-\left(\frac{\mathrm{v}}{\mathrm{c}}\right)^{2}}$,

where $t_{0}$ is the time of the static clock and $t$ is the time of the moving clock.

According to the "vk constant value principle" described in part 2 above, it can be deduced that:

$\mathrm{v}=\frac{\mathrm{v}_{0}}{\sqrt{1-\left(\frac{\mathrm{v}_{\mathrm{C}}}{\mathrm{C}}\right.}}$, where $\mathrm{v}$ is the natural frequency of the space-time quanta of the moving object, and $v_{0}$ is the natural frequency of the space-time quanta of the stationary object.

According to the $\mathrm{E}=\mathrm{h} v=\mathrm{mc}^{2}$ formula described above, the mass-increase effect of special relativity can be obtained: $\mathrm{m}=\frac{\mathrm{m}_{0}}{\sqrt{1-\left(\frac{\mathrm{v}}{\mathrm{c}}\right)^{2}}}$

In conclusion, all the basic conclusions of special relativity can be drawn on the basis of quantized space-time waves and Lorentz transformation. The difference is that special relativity is based on continuous and smooth space-time, while this paper is based on discrete quantized space-time. 


\section{Reconstructing general relativity by considering the view of quantized space-time waves}

In 1687, Newton proposed the law of universal gravitation [12]. Any two objects in the universe have an interacting gravitational force, whose magnitude is directly proportional to the mass of the two objects and inversely proportional to the square of the distance between them.

The formula is: $F=\left(G \times m_{1} \times m_{2}\right) / R^{2}$, where $G$ is the gravitational constant, $m$ is the mass of the object, and $\mathrm{R}$ is the space distance between the two objects.

Although Newton's gravitational equation was fully verified before the 20th century, the theory has its exact application scope, and it is only applicable to the weak gravitational field. Under the condition of strong gravitational field, we need to use gravitational field equation of Einstein's

general relativity [3] $\mathrm{G}_{\mathrm{uw}}=\frac{8 \pi \mathrm{G}}{\mathrm{c}^{4}} \mathrm{~T}_{\mathrm{uw}}$, where $\mathrm{G}_{\mathrm{uw}}$ is the Ricci tensor, and $\mathrm{T}_{\mathrm{uw}}$ is the energy momentum tensor representing the distribution and motion of matter. However, the equation is a second-order nonlinear partial differential equation, and it is difficult to get an exact solution. The concept of gravity was also replaced by the curvature of space-time.

Assuming that an object is launched from the surface of a celestial body with a radius of $\mathrm{R}$ and a mass of $\mathrm{M}$ at a certain speed of $\mathrm{v}$ and just escapes from the gravitational bondage of the celestial body, the kinetic energy of the object is 0 after it accidentally overcomes the gravitational potential energy of the earth. At this time, the mass of the object is $\mathrm{m}_{0}$,

According to Newton's law of gravitation: $V=\sqrt{\frac{2 \mathrm{GM}}{\mathrm{R}}}$,

According to the special relativity theory, the mass of the object has changed due to the decrease of the speed caused by overcoming the gravity of the celestial body. The energy of the object at the surface of the celestial body is:

$$
\mathrm{E}=\frac{\mathrm{m}_{0} \mathrm{c}^{2}}{\sqrt{1-\left(\frac{\mathrm{V}}{\mathrm{c}}\right)^{2}}}
$$

By combining the above two formulas, we can get: $\mathrm{E}=\frac{\mathrm{m}_{0} \mathrm{c}^{2}}{\sqrt{1-\frac{2 \mathrm{GM}}{\mathrm{Rc}^{2}}}}$

From this, we can find that the mass of the object affected by the gravitational field will be increased. Also, from the viewpoint of quantized space-time waves, it is not difficult to see that the mass-increase effect is caused by the decrease of the emission frequency of space-time quanta (time expansion effect). 
So $\mathrm{k}=\mathrm{k}_{0} \sqrt{1-\frac{2 \mathrm{GM}}{\mathrm{Rc}^{2}}}$, where $\mathrm{k}_{0}$ is the emission frequency of space-time quanta of the object at the infinite distance under the condition where the gravitational field intensity is 0 , and $\mathrm{k}$ is the emission frequency of the space-time quanta of the object at the distance $\mathrm{R}$ from the center of the celestial body after being affected by the gravity of the celestial body.

We can also get: $\mathrm{t}=\mathrm{kt}_{\mathrm{p}}=\mathrm{k}_{0} \mathrm{t}_{\mathrm{p}} \sqrt{1-\frac{2 \mathrm{GM}}{\mathrm{Rc}^{2}}}=\mathrm{t}_{0} \sqrt{1-\frac{2 \mathrm{GM}}{\mathrm{Rc}^{2}}}$, where $\mathrm{t}_{0}$ is the time interval of the object at the infinite distance where the gravitational field strength is 0 , and $t$ is the time interval of the object at the distance $\mathrm{R}$ from the center of the celestial body after being affected by the gravity of the celestial body. This formula coincides with the time expansion formula of general relativity.

It is assumed that there is an object with a mass of $\mathrm{m}_{0}$ at an infinite distance (the strength of the gravitational field is 0 ) from a celestial body with a mass of $\mathrm{M}$. Then, the object is constantly approaching the celestial body due to the gravitation, and is located at a position $\mathrm{R}$ from the spherical center of the celestial body. If the magnitude of the gravitation between them is $F$, the

work done by gravity in this process is $W=\frac{\mathrm{GMm}_{0}}{\mathrm{R}^{2}} \mathrm{R}=\mathrm{FR}$, according to Newtonian mechanics theory. Combined with $\mathrm{E}=\frac{\mathrm{m}_{0} \mathrm{c}^{2}}{\sqrt{1-\frac{2 \mathrm{GM}}{\mathrm{Rc}^{2}}}}$, the following equation can be obtained:

$\mathrm{W}=\mathrm{FR}=\frac{\mathrm{m}_{0} \mathrm{c}^{2}}{\sqrt{1-\frac{2 \mathrm{GM}}{\mathrm{Rc}^{2}}}}-\mathrm{m}_{0} \mathrm{c}^{2}$

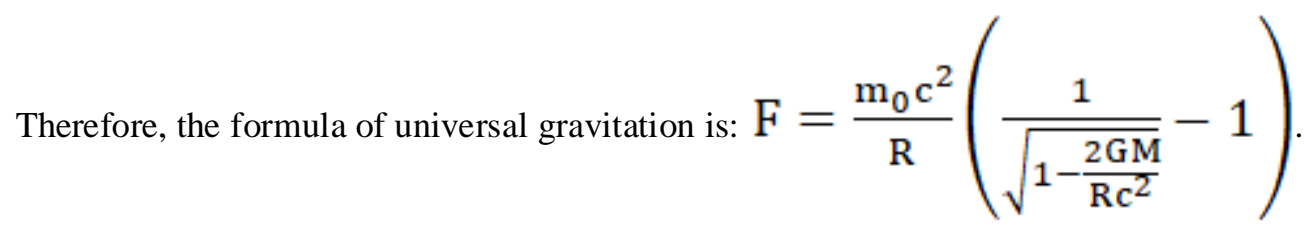

Combined with the earth's mass, radius and other parameters, it is not difficult to see that the gravity calculated by the formula under the condition of weak gravitational field is basically the same as the result calculated by the Newtonian formula. However, there are obvious prediction differences between them under the condition of strong gravitational field. 
Considering that the mass of the object at the distance $\mathrm{R}$ from the center of the celestial body is $\frac{\mathrm{m}_{0}}{\sqrt{1-\frac{2 \mathrm{GM}}{\mathrm{Rc}^{2}}}}$ the gravitational acceleration of the object is: $\mathrm{g}=\frac{\mathrm{c}^{2}}{\mathrm{R}}\left(1-\sqrt{1-\frac{2 \mathrm{GM}}{\mathrm{Rc}^{2}}}\right)$.

It can be seen from the above that the energy of the spherical gravitational field (the total energy of space-time quanta of the celestial body at a certain time point) generated instantaneously around the celestial body is $\mathrm{Mc}^{2}$, and the energy of the gravitational field contained in the particle at the distance $\mathrm{R}$ from the center of the celestial body is:

$\mathrm{E}=\frac{\mathrm{Mc}^{2}}{4 \pi \mathrm{R}^{2}}$

Furthermore, if the particle moves around the celestial body in the uniform circular motion, the space curvature $\mathrm{K}$ of the circular motion orbit of the particle is the reciprocal of the radius $\mathrm{R}$, that is, $K=1 / R$.

Combined with $\mathrm{k}=\mathrm{k}_{0} \sqrt{1-\frac{2 \mathrm{GM}}{\mathrm{Rc}^{2}}}$, we can get the following formula:

$\frac{8 \pi \mathrm{G}}{\mathrm{c}^{4}} \mathrm{E}=\mathrm{K}\left[1-\left(\frac{\mathrm{k}}{\mathrm{k}_{0}}\right)^{2}\right]$

The equation shows the change of space curvature and time expansion effect of a particle captured by the gravitational field of a celestial body with a certain mass-energy. $\frac{8 \pi G}{c^{4}}$ is a fixed coefficient, which is consistent with that of the gravitational field equation of general relativity [3] The left side of the equation represents the energy distribution of the object, while the right side represents the curvature of space-time around the object. Therefore, this equation has similar meaning to the gravitational field equation of general relativity, but it is easy to get a clear solution.

\section{Explaining dark matter and dark energy from the view of quantized space-time waves}

In 1933, Fritz Zwicky [13] discovered that the gravitational mass is far greater than the photometric mass through astronomical observations of the Coma Cluster of Galaxies, so he first proposed the concept of "dark matter" [7]. There is a large amount of dark matter that is invisible but can exert gravitational force, thus providing additional gravity to maintain the high-speed 
operation of the entire galaxy without falling apart. Later, astronomers such as Vera Rubin [14] further provided sufficient observational evidences for the existence of "dark matter". The nature of "dark matter" is still the main topic of discussion in the physics community. One theory is that "dark matter" consists of unknown elementary particles which is more common than particles that constitute the ordinary matter. However, such particles have never been directly observed by various detection methods in decades. Another theory is that there is no "dark matter" at all, and for this reason, an modified Newtonian or Einstein's theory model should be proposed to explain the formation mechanism of extra gravity.

According to the formula $\mathrm{E}=\frac{\mathrm{m}_{0} \mathrm{c}^{2}}{\sqrt{1-\frac{2 \mathrm{GM}}{\mathrm{Rc}^{2}}}}$ described above, it can be seen that the energy (E) of an object will be changed after it enters into a gravitational field environment with a certain intensity, and its increasing degree is directly proportional to the intensity of the gravitational field.

Combined with the mass-energy equation of special relativity, the following equation can be obtained:

$\mathrm{m}=\frac{\mathrm{m}_{0}}{\sqrt{1-\frac{2 \mathrm{GM}}{\mathrm{Rc}^{2}}}}$

where $m$ is the mass of the object after entering the gravitational field environment.

It can be seen that the mass of the outer objects captured by the gravitational field of the galaxy will be increased. With the gravitational collapse of the galaxy, the gravitational mass of all matter in the galaxy will increase accordingly. When the radius of the galaxy collapses from R1 to R2, the mass change of any object in this galaxy is:

$$
\Delta \mathrm{m}=\mathrm{m}_{0}\left(\frac{1}{\sqrt{1-\frac{2 \mathrm{GM}}{\mathrm{R}_{2} \mathrm{c}^{2}}}}-\frac{1}{\sqrt{1-\frac{2 \mathrm{GM}}{\mathrm{R}_{1} \mathrm{c}^{2}}}}\right)
$$

where $\mathrm{m}_{0}$ is the initial mass of the object in the gravitational field of 0 intensity, and $\mathrm{M}$ is the total mass of the galaxy.

Since the brightness of galaxies mainly comes from the luminosity of stars, and it usually takes hundreds of thousands of years for photons to be generated and moved from the core to the surface of stars, the above effects mainly describe the increase of the gravitational mass of objects. On the other hand, the effect of photometric mass increment will be delayed obviously. This explains why the photometric mass of most galaxies is smaller than the gravitational mass, and also explains the source of mass that provides additional gravity. In other words, "dark matter" 
does not exist, and its physical effects can be explained and calculated by the above model.

The above understanding of dark matter can also be used to explain another extreme situation: in the strong gravitational field environment of the accretion disk around the black hole, the time velocity is extremely slow, and the mass-energy conversion efficiency is enhanced. At this time, the energy of a single space-time quantum of the matter is extremely high which also means that its mass is extremely large. When the mass is converted into pure energy, it will release ultra-high energy photons (driven by the gravitational mass-increase effect and the mass-energy conversion effect together). At the same time, due to the extremely low emission frequency of space-time waves, the matter near the black hole radiates energy outward as extremely brief gamma-ray bursts [15]. Other similar situations, such as the huge energy generation mechanism of quasars [16], can also be explained by the model similar to the above discussion. The mathematical equation describing this process is as follows:

$$
\mathrm{E}=\mathrm{hv}=\mathrm{E}_{0} \frac{\mathrm{k}_{0}}{\mathrm{k}}
$$

where $\mathrm{E}$ is the energy of a single photon in a gamma ray burst in a strong gravitational field, $v$ is the frequency of the photon, $\mathrm{E}_{0}$ is the radiation energy of matter in a weak gravitational field, $\mathrm{k}$ is the emission frequency of space-time waves in a strong gravitational field, and $\mathrm{k}_{0}$ is the emission frequency of space-time waves in a weak gravitational field.

Next, we discuss dark energy. In 1998, two independent astronomical observation groups measured the distance between supernovae and got an astonishing discovery [17,18]: the universe is accelerating expansion, rather than the previously predicted deceleration expansion. According to Newton's theory of gravitation, all known substances in the universe produce gravitation, which leads to the tendency of mutual attraction. Therefore, the mainstream view is that the universe is full of "dark energy" with negative pressure, which produces repulsive effect and drives the accelerating expansion of the universe. "Dark energy" is similar to "dark matter", which does not participate in electromagnetic interaction and is evenly distributed in the universe. With the expansion of the universe, its total amount is increasing and its repulsion effect is becoming stronger and stronger. According to statistics, "dark energy" accounts for about $65 \%$ of the total amount of matter in the universe. It is also believed that "dark energy" does not exist, but we need to modify Newton / Einstein's gravitational field equation.

According to the above calculation, when the radius of the universe expands from $\mathrm{R} 1$ to $\mathrm{R} 2$, the mass change trend of any object in the universe is just the opposite to that in the collapse, and the calculation formula describing the mass reduction degree is as follows:

$$
\Delta \mathrm{m}=\mathrm{m}_{0}\left(\frac{1}{\sqrt{1-\frac{2 \mathrm{GM}}{\mathrm{R}_{1} \mathrm{c}^{2}}}}-\frac{1}{\sqrt{1-\frac{2 \mathrm{GM}}{\mathrm{R}_{2} \mathrm{c}^{2}}}}\right)
$$


According to the mass-energy equation, the energy reduction value is:

$$
\Delta \mathrm{E}=\mathrm{m}_{0} \mathrm{c}^{2}\left(\frac{1}{\sqrt{1-\frac{2 \mathrm{GM}}{\mathrm{R}_{1} \mathrm{c}^{2}}}}-\frac{1}{\sqrt{1-\frac{2 \mathrm{GM}}{\mathrm{R}_{2} \mathrm{c}^{2}}}}\right)
$$

where $\mathrm{M}$ is the total mass of the universe.

It is assumed that an observer continuously observes the regression speed of a galaxy at a distance $\mathrm{D}$ and finds that the speed increases from V1 to V2 when the radius of the universe expands from $\mathrm{R} 1$ to $\mathrm{R} 2$. The initial mass of the galaxy is $\mathrm{m}_{0}$, and the total mass of the universe is $\mathrm{M}$. Combined with Hubble's law, there is the following relationship:

$$
\begin{aligned}
& \frac{\mathrm{m}_{0} \mathrm{c}^{2}}{\sqrt{1-\frac{2 \mathrm{GM}}{\mathrm{R}_{1} \mathrm{c}^{2}}}}-\frac{\mathrm{m}_{0} \mathrm{c}^{2}}{\sqrt{1-\frac{2 \mathrm{GM}}{\mathrm{R}_{2} \mathrm{c}^{2}}}}=\frac{\mathrm{m}_{0} \mathrm{c}^{2}}{\sqrt{1-\left(\frac{\mathrm{v}_{2}}{\mathrm{c}}\right)^{2}}}-\frac{\mathrm{m}_{0} \mathrm{c}^{2}}{\sqrt{1-\left(\frac{\mathrm{v}_{1}}{\mathrm{c}}\right)^{2}}} \\
& =\frac{\mathrm{m}_{0} \mathrm{c}^{2}}{\sqrt{1-\left(\frac{\mathrm{H}_{2} \mathrm{D}}{\mathrm{c}}\right)^{2}}}-\frac{\mathrm{m}_{0} \mathrm{c}^{2}}{\sqrt{1-\left(\frac{\mathrm{H}_{1} \mathrm{D}}{\mathrm{c}}\right)^{2}}},
\end{aligned}
$$

After simplification, the relationship between Hubble constant and cosmic radius is obtained:

$$
\left(\frac{\mathrm{H}_{1}}{\mathrm{H}_{2}}\right)^{2}=\frac{\mathrm{R}_{1}}{\mathrm{R}_{2}}
$$

In addition, $\mathrm{H}=\sqrt{\mathrm{R} \Lambda}$, where $\Lambda$ is a constant number related to the space-time structure of the universe.

It can be seen that the expansion of the universe will cause the loss of mass / energy of matter, which can be converted into "dark energy". According to the above formulas, with the increase of the cosmic radius scale, the dark energy and Hubble constant $\mathrm{H}$ are getting larger and larger, thus accelerating the expansion of the universe. The changing trend is consistent with the actual situation.

\section{Prediction of the universe evolution from the view of quantized space-time waves}

Since 1965, modern astronomy believes that the universe was born from the "big bang singularity" 13.8 billion years ago $[19,20]$. At the earliest moment of its birth, it experienced a short period of "inflation", followed by a period of decelerating expansion. Six billion years ago, the universe 
went from decelerating expansion to accelerating expansion, and has remained that way ever since. There are probably two possibilities for the future destiny of the universe: one is to continue to expand, and the other is to contract so that it collapses into a singularity. Whether it expands or contracts is thought to depend on the average density of the universe. If the density of matter in the universe is less than the critical density, the universe will keep expanding; and if the density of matter is greater than the critical density, the expansion of the universe will be slower and slower until it contracts back into a singularity. Theories based on dark energy suggest that the universe will continue to expand and end in a big rip. Whether and when the big rip will happen is still widely discussed.

It can be seen from the above description that the emission frequency $(\mathrm{k})$ of space-time waves will be decreased for objects moving at a high speed or in a strong gravitational field, in other words, the time passing rate around the objects will be slowed down. The emission frequency of space-time waves of matter at the speed of light or in the space-time singularity is 0 , that is to say, the observer will not feel any gravitational effect from the matter, rather than that the mass-energy of the object predicted by special relativity will reach infinity, nor that the curvature of space-time predicted by general relativity will be infinity.

From the viewpoint of quantized space-time waves, the product (vk) of the natural frequency (v) of space-time waves of all matters and the emission frequency $(\mathrm{k})$ of the space-time quanta is a certain value in the evolution of the universe. Therefore, the natural frequency (v) of the space-time waves reaches the maximum value $v_{\max }$, while the emission frequency $(\mathrm{k})$ reaches the minimum value $\mathrm{k}_{\min }$ at the moment before the extreme conditions at the singularity are formed. At the singularity, $v=0$ and $k=0$, which means that the singularity has no physical meaning. In other words, the singularity does not actually exist. After the singularity, in order to keep vk as a certain value, $\mathrm{k}$ will reach the maximum value $\mathrm{k}_{\max }$ in an instant, while $v$ is at the minimum value $\mathrm{v}_{\min }$ instantaneously. The infinite increase of $\mathrm{k}$ value will lead to the instantaneous inflation of the whole space-time. It can be seen that the existing universe originated from the big bang of the last universe. The mathematical expression of the above process is: $v_{\max } \mathrm{k}_{\min }=v_{\min } \mathrm{k}_{\max }$.

Let's consider the opposite extreme case: assuming that the universe is constantly expanding and approaching the limit "big rip " under the promotion of increasing dark energy. At this time, the emission frequency $(\mathrm{k})$ of space-time waves reaches the maximum value $\mathrm{k}_{\max }$, and the natural frequency reaches the minimum value $v_{\min }$. At the big rip, $v=0$ and $\mathrm{k}=0$, which means that the big rip does not have actual physical meaning. In other words, it does not exist. After that, in order to keep $v \mathrm{k}$ as a certain value, $v$ will reach the maximum value $v_{\max }$ in an instant, while $\mathrm{k}$ will be at the minimum value $k_{\min }$ instantaneously. The infinite decrease of $\mathrm{k}$ value will cause the instantaneous collapse of the whole space-time. The mathematical equation of the above process is: $v_{\min } \mathrm{k}_{\max }=v_{\max } \mathrm{k}_{\min }$. It can be seen that the future universe will not end in the great rip, but will collapse again.

The above discussion is consistent with the principle of "extremes of things must be reversed" in ancient Chinese philosophy.

After resolving the above two extremes, we can also roughly infer the past and future of the universe: There is an inflation at the beginning of the existing universe. With the continuous emergence of matter, gravity gradually plays the role of cohesion, which slowed down the 
expansion of the universe. However, with the continuous expansion of the universe, the mass-energy of matter is decreased, and the "dark energy" is increased gradually and accelerates the expansion of the universe again. When the infinite expansion of space is close to the big rip, it will instead collapse. Then, with the reappearance of "dark energy", the repulsive force gradually plays the role of exclusion, which slows down the universe collapse. However, with the continuously collapse of the universe, the mass-energy of matter is increased, and "dark matter" gradually takes the upper hand and overcomes the role of space expansion, therefore accelerating space collapse again until the next big bang.

\section{Conclusion}

Based on the heuristic view of quantized space-time waves, this paper establishes a new space-time model of the universe, thus providing a preliminary unified framework for explaining the difficult problems in modern physics, such as dark matter, dark energy, gamma-ray burst, high energy emission mechanisms in quasar, and the origin and evolution of the universe. This is only an outline of the work, the future related theoretical issues are needed to be discussed and analyzed in depth.

\section{References}

[1]A. Einstein, "Zur Elektrodynamik bewegter Körper," Ann. Phys. 17, 891-921 (1905).

[2] H.Minkowski, "Raum und Zeit, " Physikalische Zeitschrift 10, 104-111 (1909).

[3]A. Einstein, "Die Grundlage der allgemeinen Relativitätstheorie," Ann. Phys. 49, 769-822 (1916).

[4]K. Schwarzschild, "Uber das Gravitationsfeld eines massenpunktes nack der Einsteinschen Theorie”, Sitz. Deut. Akad. Wiss. Berlin, KI. Math. - Phys. Tech., 189 - 196 (1916).

[5]G. Lemaître, "Un univers homogène de masse constante et de rayon croissant, rendant compte de la vitesse radiale des nébuleuses extra-galactiques". Annales de la Société scientifique de Bruxelles, Série A, 47, 49-59 (1927).

[6]A.Zeilinger, "A FOUNDATIONAL PRINCIPLE FOR QUANTUM MECHANICS". Foundations of Physics, 29(4): 631-643(1999).

[7] G. Bertone, D. Hooper, J. Silk, et al. "Particle dark matter: Evidence, candidates and constraints". Physics Reports, 405(5): 279-390(2005).

[8] E. J. Copeland, M. Sami, S. Tsujikawa, et al. "Dynamics of dark energy". International Journal of Modern Physics D, 15(11): 1753-1935(2006).

[9] C. Rovelli, L. Smolin. "Loop space representation of quantum general relativity". Nuclear Physics, 331(1): 80-1529(1990).

[10] T. W. Noonan. "The validity of the formula $E=m c^{2}$ for gravitational energy". General Relativity \& Gravitation, 19(1):47-56(1987).

[11] H.A.Lorentz, "Electromagnetic phenomena in a system moving with any velocity less 
than that of light". Proc. Acad. Sci. Amsterdam 6, 809 (1904).

[12]I.Newton ,"Philosophiae Naturalis Principia Mathematica" ("Mathematical Principles of Natural Philosophy"), London (1687).

[13] F.Zwicky, "Die Rotverschiebung von extragalaktischen Nebeln". Helvetica Physica Acta, 6, 110-127(1933).

[14] V. C. Rubin. "The rotation of spiral galaxies". Science, 220(4604): 1339-1344(1983).

[15]T. Piran. "The physics of gamma-ray bursts". Reviews of Modern Physics, 76(4): 1143-1210(2005).

[16].D. Lynden-Bell. "Galactic nuclei as collapsed old quasars". Nature, 223: 690-694(1969).

[17].A.G.Riess, et al."Observational evidence from supernovae for an accelerating universe and a cosmological constant". Astron. J. 116, 1009 - 1038 (1998).

[18].S.Perlmutter, et al. Measurements of omega and lambda from 42 high-redshift supernovae. Astrophys. J. 517, 565-586 (1999).

[19].A. Feinstein, K. E. Kunze, M. A. Vazquezmozo, et al. "Initial conditions and the structure of the singularity in pre - big bang cosmology". Classical and Quantum Gravity, 17(18): 3599-3616(2000).

[20]. P. Coles. The end of the old model Universe. Nature, 393(6687): 741-744(1998). 